\title{
On edge states in semi-infinite quantum Hall systems
}

\author{
N Macrisł $\dagger$, P A Martin and J V Puléł \\ $\dagger$ Institut de Physique Theorique, Ecole Polytechnique Federale de Lausanne, CH-1015 \\ Lausanne, Switzerland \\ ¥Department of Mathematical Physics, University College Dublin, Belfield 4, Dublin, Ireland
}

Received 8 October 1998

\begin{abstract}
We consider an electron in two dimensions submitted to a magnetic field and to the potential of impurities. We show that when the electron is confined to a half-space by a planar wall described by a smooth increasing potential, the total Hamiltonian necessarily has a continuous spectrum in some intervals in between the Landau levels provided that both the amplitude and spatial variation of the impurity potential are sufficiently weak. The spatial decay of the impurity potential is not needed. In particular, this proves the occurrence of edge states in semi-infinite quantum Hall systems.
\end{abstract}

\section{Introduction}

In the quantum Hall effect the physics at the boundary of the sample plays an important role. This was first recognized in the context of the integer effect by Halperin [1], and more recently has been the subject of the powerful and beautiful theories of the fractional effect [2-4]. Moreover, the edge properties of quantum Hall fluids are now accessible experimentally [5] and provide much information on these systems. Halperin argued that in a two-dimensional system with boundaries (say an annulus or a cylinder) submitted to a perpendicular magnetic field there are 'quasi-one-dimensional edge states' extended along each boundary of the sample, which contribute to the quantized Hall conductivity (if the chemical potentials on opposite edges are different). In the ideal situation of a clean sample with non-interacting electrons it is easy to construct such quasi-one-dimensional edge states (see equation (1.6) below). In a real situation, however, the sample is disordered (we disregard Coulomb interactions here) and the existence of extended states may seem less evident since usual one-dimensional systems exhibit localization for any amount of disorder. In fact, the absence of localization in this situation is usually explained by the chirality of the modes propagating along the boundary. The simplest models for investigating the boundary of quantum Hall fluids are constructed from one-dimensional electron systems with chiral branches of excitations (see, for example, $[2,4]$ ) corresponding to only left-movers (or only right-movers). In such systems the absence of interference between left- and right-movers leads to the absence of Anderson localization and hence to extended states.

In the present work we consider a two-dimensional semi-infinite geometry, the electrons being confined by a planar smooth wall. Starting right away from an unbounded system allows a clear-cut distinction between a point and a continuous spectrum in the Schrödinger operator. We show here that for a large class of additional impurity potentials (random or deterministic) the Hamiltonian of this system does indeed have continuous parts in its spectrum as a result of the presence of the wall. We recall that for the case of infinite space (system without boundary) 
and point random impurities, the spectrum has been shown to be entirely dense pure point for a large enough magnetic field [6], a fact which is believed to hold for more general random potentials $\dagger$. For these, existing results deal only with limited regions of the spectrum [7-9]. Here we are mainly concerned with the opposite problem, namely the exclusion of a point spectrum in certain parts of the spectrum $\ddagger$. During the completion of this work, we have been informed that Froehlich, Graf and Walcher [10] have proven the existence of intervals of absolute continuity for the same system with the help of Mourre estimates, and have also treated the case of Dirichlet boundary conditions.

The position of the electron in the plane is $r=(x, y)$, where $y$ is the direction parallel to the wall. We assume that the 'wall potential' $U(x)$ vanishes for $x \leqslant 0$ (the bulk region) and increases as a power law for $x>0$ (the wall region)

$$
U(x)=0 \quad x \leqslant 0 \quad U(x)=\mu x^{\gamma} \quad x \geqslant 0
$$

where $\mu>0$ and $\gamma \geqslant 1$. All our estimates hold for wall potentials that grow much faster as long as they are continuous on the whole real line and strictly increasing (for example, exponential or Gaussian growth), but we limit ourselves to (1.1) for the sake of concreteness.

In addition, the electron is also submitted to a bounded and differentiable external potential $w(\boldsymbol{r})$ such that

$$
\sup _{r}|w(\boldsymbol{r})|=w_{0}<\infty \quad \sup _{\boldsymbol{r}}\left|\partial_{x} w(\boldsymbol{r})\right|=w_{0}^{\prime}<\infty .
$$

The total Hamiltonian is

$$
H=H_{0}+w(\boldsymbol{r})
$$

where $H_{0}$ is the pure wall Hamiltonian

$$
H_{0}=H(B)+U(x)
$$

and

$$
H(B)=\frac{1}{2} p_{x}^{2}+\frac{1}{2}\left(p_{y}-B x\right)^{2}
$$

is the usual Landau Hamiltonian written in the Landau gauge, where $B$ is the strength of the magnetic field. All these Hamiltonians are essentially self-adjoint on $C_{0}^{\infty}\left(\mathbb{R}^{2}\right)$ ([11], theorem X34, p 190).

It is well known that the spectrum of $H(B)$ consists of Landau levels $E_{n}=\left(n+\frac{1}{2}\right) B$, $n=0,1,2, \ldots$ These are infinitely degenerate so that one may construct localized as well as extended eigenfunctions of $H(B)$ by appropriate linear combinations. Because of translation invariance in the $y$-direction the generalized eigenfunctions of $H_{0}$ are of the form

$$
\mathrm{e}^{\mathrm{i} k y} h_{n k}(x)
$$

where $h_{n k}(x)$ is the solution of the one-dimensional problem $\mathcal{H}(k) h_{n k}=\mathcal{E}_{n}(k) h_{n k}$,

$$
\mathcal{H}(k)=\frac{1}{2} p_{x}^{2}+\frac{1}{2}(k-B x)^{2}+U(x) .
$$

For a given $k$ this is the Hamiltonian of a particle in a confining potential well for both $x \rightarrow \pm \infty$. Therefore, the spectrum consists of non-degenerate eigenvalues $\mathcal{E}_{n}(k)$ and it follows from the Kato-Rellich theorem [12] that the branches $\mathcal{E}_{n}(k)$ are analytic functions of $k$.

Note also that from the Feynman-Hellman theorem

$$
\begin{aligned}
\partial_{k} \mathcal{E}_{n}(k) & =\int_{-\infty}^{+\infty} \mathrm{d} x(k-B x)\left|h_{n k}(x)\right|^{2} \\
& =\frac{1}{B} \int_{-\infty}^{+\infty} \mathrm{d} x U^{\prime}(x)\left|h_{n k}(x)\right|^{2} .
\end{aligned}
$$

$\dagger$ This is compatible with a localization length which may diverge at isolated energies.

$\ddagger$ We do not exclude the possibility of a singular continuous spectrum. 
Since $U^{\prime}(x)>0$ for $x>0$ the branches are increasing strictly monotonically for all $k$. The equality $(1.8 b)$ follows from the fact that the Hamiltonian (1.7) is unitarily equivalent to

$$
\frac{1}{2} p_{x}^{2}+\frac{1}{2} B^{2} x^{2}+U\left(x+\frac{k}{B}\right) .
$$

Moreover, $\lim _{k \rightarrow-\infty} \mathcal{E}_{n}(k)=\left(n+\frac{1}{2}\right) B$ since $U(x+k / B)$ vanishes in this limit, whereas $\lim _{k \rightarrow+\infty} \mathcal{E}_{n}(k)=+\infty$ since $U(x+k / B)$ is unbounded as $k \rightarrow+\infty$. One concludes from these observations that the spectrum of the wall Hamiltonian $H_{0}$ is absolutely continuous and consists of the set $\sigma\left(H_{0}\right)=[B / 2,+\infty[$.

The right-hand side of (1.8a) is the average of the diamagnetic current $j_{y}=p_{y}-B x$ carried by the state (1.6) along the wall and $\partial_{k} \mathcal{E}_{n}(k)$ can be interpreted as the corresponding group velocity.

The main question that we address in this paper is the stability of this continuous spectrum (or parts of it) when an impurity potential $w$ is added to $H_{0}$. Consider first the case when $w$ is localized in a finite region of space. Then, as shown in appendix $\mathrm{A}, \mathrm{e}^{-t H}-\mathrm{e}^{-t H_{0}}$ is a trace class operator, implying that $H$ still has an absolutely continuous spectrum in [ $B / 2,+\infty[$ ( [13], chapter X). If $w$ does not decay at infinity, as is the case, for example, for periodic or random potentials, this argument cannot be applied and the nature of the spectrum may be different. In the random case a convenient model of disorder is obtained by assuming that the impurities are located on a regular lattice, each of them being the source of the same local bounded potential, with bounded derivatives. Then for $w(\boldsymbol{r})$ we take a typical realization of the random potential

$$
V_{\omega}(\boldsymbol{r})=\sum_{(n, m) \in \mathbb{Z}^{2}} \omega_{n, m} v(x-n, y-m) \quad v(\boldsymbol{r})=0 \quad \text { for } \quad|\boldsymbol{r}| \geqslant \frac{1}{2}
$$

where $\omega_{n, m}$ are independent identically distributed random variables with continuous density supported in $[-1,1]$. If $v(\boldsymbol{r})$ satisfies (1.2) the same is true for all $V_{\omega}(\boldsymbol{r})$. Typical realizations of the random potential do not decay at large distance, so that trace class perturbation theorems cannot be applied.

We prove in section 4 , theorem 1 , that for $w_{0}$ and $w_{0}^{\prime}$ small enough, depending on $B$ and the steepness of the wall, $H$ cannot have a point spectrum in intervals $\Delta_{n}(B, \delta)=$ ]$(n+1) B-\delta,(n+1) B+\delta$ [ of size $2 \delta>B-w_{0}$ in between the Landau levels. This information is supplemented in section 5 , theorem 2 , by a proof that the intervals $\Delta_{n}(B, \delta)$ are included in the spectrum of $H$. Hence theorems 1 and 2 imply that the spectrum of $H$ remains continuous in these intervals. In the random case, general arguments (appendix B) show that the spectrum of $H_{\omega}$ contains $[B / 2, \infty$ [ with probability one, and thus theorem 1 alone implies the existence of a continuous spectrum for almost all $\omega$. Theorem 2 guarantees the existence of a continuous spectrum for all realizations of (1.10) and allows us to treat deterministic potentials (e.g. periodic).

\section{Main results}

In the following sections, the effect of the wall will enter mainly through the following functional of the wall potential:

$$
A(E ; U)=\sup _{0 \leqslant x \leqslant x_{0}(U)}\left(\frac{U(x)^{4}}{U^{\prime}(x)}\right)+8 \int_{x_{0}(U)}^{\infty} \mathrm{d} x \frac{U(x)^{4} U(x / 2)^{1 / 4}}{(2 \pi x)^{1 / 2} U^{\prime}(x)} \exp \left(-\frac{x}{2 \sqrt{2}} U(x / 2)^{1 / 2}\right)
$$


where $E>0$ and $x_{0}(U)$ solves $U\left(x_{0} / 2\right)=2 E$. We remark that $A(E ; U)$ is finite for a much larger class of wall potentials than those in (1.1) (e.g. exponential). The factor

$$
\exp \left(-\frac{x}{2 \sqrt{2}} U(x / 2)^{1 / 2}\right)
$$

is similar to the WKB tail of the wavefunction $\exp \left(-\int^{x} \mathrm{~d} y \sqrt{U(y)-E}\right) \sim \exp (-c x \sqrt{U(x)})$ in the wall region. We have

Theorem 1. If $B / 2-w_{0}>\delta$ for some $\delta>0$ and

$$
w_{0}^{\prime}<\frac{\left(B / 2-\delta-w_{0}\right)^{4}}{\sup _{E \in \Delta_{n}(B, \delta)} A\left(E+w_{0} ; U\right)}
$$

then $H$ has no eigenvalue in the intervals $\left.\Delta_{n}(B, \delta)=\right](n+1) B-\delta,(n+1) B+\delta[$.

Theorem 2. If $B / 2-w_{0}>\delta$ for some $\delta>0$ and

$$
w_{0}^{\prime}<\frac{\left(B / 2-\delta-w_{0}\right)^{4}}{\sup _{0 \leqslant w_{0} \leqslant B / 2} \sup _{E \in \Delta_{n}(B, \delta)} A\left(E+w_{0} ; U\right)}
$$

then the whole interval $\Delta_{n}(B, \delta)$ is included in the spectrum of $H$.

Note that condition (2.3) implies (2.2), hence under condition (2.3) the spectrum of $H$ in $\Delta_{n}(B, \delta)$ is purely continuous.

In order to understand the meaning of this condition more explicitly, let us consider for concreteness the case of a 'linear wall', $U(x)=\mu x, x \geqslant 0$ and $U(x)=0, x \leqslant 0$ with $\mu>0$. Then we have

$$
A_{\text {linear }}(E)=\frac{(4 E)^{4}}{\mu}+\frac{8}{\sqrt{2 \pi} 2^{14}} \mu^{5 / 3} \int_{4 E / \mu^{2 / 3}}^{\infty} \mathrm{d} x x^{15 / 4} \mathrm{e}^{-\frac{1}{4} x^{3 / 2}} .
$$

Consider first the dependence on the steepness $\mu$ of the wall for fixed $B$ and $n$. We find

$$
w_{0}^{\prime}<\text { Cst } \mu \quad \mu \rightarrow 0 .
$$

On the other hand for a steep wall, $\mu \rightarrow \infty$, we find the condition

$$
w_{0}^{\prime}<\text { Cst } \mu^{-5 / 3} \quad \mu \rightarrow+\infty .
$$

The case (2.5) shows that our theorems can only prove the occurrence of a continuous spectrum if the wall is steep enough. For a localized impurity $(w(r)$ with compact support) we know that the spectrum of $H$ has a continuous part for any $\mu>0$ (see the introduction). When the impurities are extended over the whole space it is not known whether the same is true, or whether a critical steepness is needed for the occurrence of a continuous spectrum. On the other hand, because of (2.6) we cannot conclude about the existence of a continuous spectrum for a very steep wall. We nevertheless believe that the system still has extended edge states in the limit of hard walls. The use of nonlinear walls in (2.1) (e.g. polynomial, exponential, etc) leads to the same conclusion that an upper bound on $w_{0}^{\prime}$ has to vanish in the limit of infinite steepness. We feel that this inability to prove the existence of edge states for very steep walls is linked to the techniques used in this paper.

We consider now $B$ large and $\mu, n$ fixed. Then one can allow $w_{0}$ to be large but $w_{0}^{\prime}$ has to remain bounded because it follows from (2.3) and (2.4) that $w_{0}^{\prime} \leqslant C, C>0$ as $B \rightarrow \infty$. However, for a nonlinear wall $U(x)=\mu x^{\gamma}, x>0$ with $\gamma>1$ the latter bound can be improved to $w_{0}^{\prime} \leqslant C B^{1-1 / \gamma}$, allowing for more rapidly varying impurity potentials.

Finally, the high-energy (i.e. large- $n$ ) behaviour of the bound (2.3) is as $n^{-4}$. Thus theorems 1 and 2 guarantee the existence of a continuous spectrum only in a finite number of 
intervals. We expect, however, to find a continuous spectrum in between all Landau levels, especially at high energies. This is probably again an artefact of our methods.

To conclude this section, we say a few words about the ideas involved in the proof of theorems 1 and 2 . The absolute continuity of $\sigma\left(H_{0}\right)$ is intimately linked to the positive group velocity $\partial_{k} \mathcal{E}_{n}(k)$ and positive 'local drift' $U^{\prime}(x) / B$ in $(1.8 b)$. A closely related fact is the existence of a positive commutator of $H_{0}$ with the observable

$$
Y=p_{x}-B y
$$

which represents the centre of the cyclotronic orbit for the Landau problem

$$
\left[\mathrm{i} Y, H_{0}\right]=U^{\prime}(x) \geqslant 0 .
$$

Suppose there exists an eigenfunction $\Psi_{0}$ of $H_{0}$. Then

$$
0=\left\langle\Psi_{0},\left[\mathrm{i} Y, H_{0}\right] \Psi_{0}\right\rangle=\int_{0}^{+\infty} \mathrm{d} x \int_{-\infty}^{+\infty} \mathrm{d} y U^{\prime}(x)\left|\Psi_{0}(x, y)\right|^{2}
$$

which is clearly impossible because $\Psi_{0}$ has a tail penetrating the region $x>0$, where $U^{\prime}(x)$ is strictly positive. This observation can be generalized to the full Hamiltonian (2.3). The commutator with $Y=p_{x}-B y$ becomes

$$
[\mathrm{i} Y, H]=U^{\prime}(x)+\partial_{x} w(\boldsymbol{r}) .
$$

Now we lose the positivity because of $\partial_{x} w$, but we may exploit the fact that $\partial_{x} w$ is bounded and $U^{\prime}(x) \rightarrow+\infty$ for $x \rightarrow+\infty$. If $H$ has an eigenstate $\Psi$, then $\langle\Psi,[\mathrm{i} Y, H] \Psi\rangle=0$ so that

$$
\left\langle\Psi, U^{\prime} \Psi\right\rangle=-\left\langle\Psi, \partial_{x} w \Psi\right\rangle \leqslant w_{0}^{\prime} .
$$

Suppose now that the eigenenergy of $\Psi$ lies in between two Landau levels, then $\Psi$ should be supported in regions where $U(x)$ is large. Indeed, if $\Psi$ were essentially localized in the bulk region, the wall would not contribute to the energy which would then lie in the vicinity of a Landau level for small $w_{0}$. Therefore, $\left\langle\Psi, U^{\prime} \Psi\right\rangle$ should be large, which contradicts (2.10) if $w_{0}^{\prime}$ is sufficiently small.

The basic idea behind the proof of theorem 2 is as follows. Suppose that $E \in \Delta_{n}(B, \delta)$ belongs to the resolvent set of $H$. Then there is a small gap around $E$ in the spectrum of $H$. It is possible to add a local perturbation to $H$ such that: (a) theorem 1 is still applicable to the perturbed Hamiltonian; (b) the local perturbation creates eigenvalues inside the gap. From (a) and (b) we obtain a contradiction so that $E \in \Delta_{n}(B, \delta)$ cannot belong to the resolvent set of $H$.

\section{Pointwise estimate of wavefunctions}

In this section we provide a control on the decay of the eigenfunctions of $H$ for $x>0$ using Brownian motion techniques. Let $\boldsymbol{b}(s)=\left(b_{x}(s), b_{y}(s)\right)$ be a two-dimensional Brownian path with $0 \leqslant s \leqslant t$ and $\boldsymbol{b}(0)=0$. We denote by $D \boldsymbol{b}=D b_{x} D b_{y}$ the Wiener measure with covariance $\left\langle b_{i}(s) b_{j}(s)\right\rangle=\min (s, t) \delta_{i j}, i, j=x, y$. For an eigenfunction $\Psi$ with eigenvalue $E$ we have, using the Feynman-Kac-Ito representation [14],

$$
\begin{aligned}
\mathrm{e}^{-t E} \Psi(\boldsymbol{r})= & \int \boldsymbol{b} \exp \left[-\mathrm{i} B \int_{0}^{t}\left(x+b_{x}(s)\right) \mathrm{d} b_{y}\right] \\
& \times \exp \left[-\int_{0}^{t} \mathrm{~d} s\left(U\left(x+b_{x}(s)\right)+w(\boldsymbol{r}+\boldsymbol{b}(s))\right)\right] \Psi(\boldsymbol{r}+\boldsymbol{b}(t)) .
\end{aligned}
$$


Using the Schwarz inequality on the measure $D b$, hypothesis (1.2), and then integrating over the $y$-direction, we obtain

$\mathrm{e}^{-2 t E} \int_{-\infty}^{+\infty} \mathrm{d} y|\Psi(\boldsymbol{r})|^{2} \leqslant \mathrm{e}^{2 t w_{0}} \int D b_{x} \exp \left[-2 \int_{0}^{t} \mathrm{~d} s U\left(x+b_{x}(s)\right)\right] \int D b_{x} F\left(x+b_{x}(t)\right)$

where

$$
F\left(x+b_{x}(t)\right)=\int_{-\infty}^{+\infty} \mathrm{d} y\left|\Psi\left(x+b_{x}(t), y\right)\right|^{2} .
$$

We have

$$
\begin{aligned}
\int D b_{x} F\left(x+b_{x}(t)\right) & =\int_{-\infty}^{+\infty} \mathrm{d} x^{\prime} \frac{\mathrm{e}^{-x^{\prime 2} / 2 t}}{(2 \pi t)^{1 / 2}} F\left(x+x^{\prime}\right) \leqslant \frac{1}{(2 \pi t)^{1 / 2}} \int_{-\infty}^{+\infty} \mathrm{d} x^{\prime} F\left(x^{\prime}\right) \\
& =\frac{1}{(2 \pi t)^{1 / 2}} \int \mathrm{d} \boldsymbol{r}|\Psi(\boldsymbol{r})|^{2}=\frac{1}{(2 \pi t)^{1 / 2}} .
\end{aligned}
$$

From (3.2) and (3.4) we have the estimate

$$
\int_{-\infty}^{+\infty} \mathrm{d} y|\Psi(\boldsymbol{r})|^{2} \leqslant \frac{\mathrm{e}^{2 t\left(E+w_{0}\right)}}{(2 \pi t)^{1 / 2}} \int D b_{x} \exp \left[-2 \int_{0}^{t} \mathrm{~d} s U\left(x+b_{x}(s)\right)\right] .
$$

Now set $x>0$. We decompose the Brownian integral in (4.5) over the two sets corresponding to long and short paths: $\Delta_{l}(x)=\left\{b_{x}\left|\sup _{0 \leqslant s \leqslant t}\right| b_{x}(s) \mid>x / 2\right\}$ and $\Delta_{s}(x)=$ $\left\{b_{x}\left|\sup _{0 \leqslant s \leqslant t}\right| b_{x}(s) \mid<x / 2\right\}$. If $b_{x} \in \Delta_{s}(x)$ we have $x+b_{x}(s)>x / 2$ for all $s$ so that $U\left(x+b_{x}(s)\right) \geqslant U(x / 2)$ and

$$
\int_{\Delta_{s}(x)} D b_{x} \exp \left[-2 \int_{0}^{t} \mathrm{~d} s U\left(x+b_{x}(s)\right)\right] \leqslant \mathrm{e}^{-2 t U(x / 2)} .
$$

On the other hand, if $b_{x} \in \Delta_{l}(x)$ we use $U\left(x+b_{x}(s)\right) \geqslant 0$ and therefore

$$
\begin{aligned}
\int_{\Delta_{l}(x)} D b_{x} \exp \left[-2 \int_{0}^{t} \mathrm{~d} s U\left(x+b_{x}(s)\right)\right] & \leqslant \int_{\Delta_{l}(x)} D b_{x} \\
& \leqslant 2 \int_{b_{x}(t) \geqslant x} D b_{x} \leqslant 2 \sqrt{2} \mathrm{e}^{-x^{2} / 4 t} .
\end{aligned}
$$

In equation (3.7) the second estimate is Levy's inequality (see [14], p 65). From equations (3.5)-(3.7) we obtain for $x>0$

$$
\int_{-\infty}^{+\infty} \mathrm{d} y|\Psi(\boldsymbol{r})|^{2} \leqslant \frac{\mathrm{e}^{2 t\left(E+w_{0}\right)}}{(2 \pi t)^{1 / 2}}\left(2 \sqrt{2} \mathrm{e}^{-x^{2} / 4 t}+\mathrm{e}^{-2 t U(x / 2)}\right) .
$$

In equation (3.8) we are still free to choose $t>0$ as we wish. With $t=(x / 2 \sqrt{2}) U(x / 2)^{-1 / 2}$ the two exponential terms in the bracket of (4.8) become equal

$\int_{-\infty}^{+\infty} \mathrm{d} y|\Psi(\boldsymbol{r})|^{2} \leqslant 8 \frac{U(x / 2)^{1 / 4}}{(2 \pi x)^{1 / 2}} \exp \left(\left(E+w_{0}\right) \frac{x}{\sqrt{2}} U(x / 2)^{-1 / 2}-\frac{x}{\sqrt{2}} U(x / 2)^{1 / 2}\right)$.

Let $x_{0}(U)$ be the solution of

$$
U\left(x_{0} / 2\right)=2\left(E+w_{0}\right) .
$$

For $x>x_{0}(U)$ the estimate (4.9) becomes

$$
\int_{-\infty}^{+\infty} \mathrm{d} y|\Psi(\boldsymbol{r})|^{2} \leqslant 8 \frac{U(x / 2)^{1 / 4}}{(2 \pi x)^{1 / 2}} \exp \left(-\frac{x}{2 \sqrt{2}} U(x / 2)^{1 / 2}\right) .
$$

This bound does not depend on the magnetic field. It merely estimates the probability density of finding the quantum particle in the classically forbidden region by the wall. 


\section{Absence of eigenvalues in between Landau levels}

The proof of theorem 1 is based on the following two lemmas.

Lemma 1. Let $\Psi$ be a normalized eigenfunction of $H$, then

$$
\left\langle\Psi,\left(U^{\prime}+\partial_{x} w(x, y)\right) \Psi\right\rangle=0 .
$$

The relation (4.1) follows formally from (2.9), but requires a proof since $Y$ is an unbounded function of the $y$ coordinate. The proof is given in appendix $\mathrm{C}$.

Lemma 2. Let $B / 2-w_{0}>\delta>0$ and $\Psi$ be a normalized eigenfunction of $H$ with eigenvalue $E \in \Delta_{n}(B, \delta)$. Then

$$
\|U \Psi\| \geqslant B / 2-\delta-w_{0} .
$$

Proof. By hypothesis $\operatorname{dist}\left(E, \sigma(H(B)) \geqslant B / 2-\delta\right.$, therefore $(H(B)-E)^{2} \geqslant(B / 2-\delta)^{2}$, which implies

$$
\|(H(B)-E) \Psi\| \geqslant(B / 2-\delta)\|\Psi\|=B / 2-\delta .
$$

Moreover, since $(H(B)-E) \Psi=-(U+w) \Psi$ we have

$$
B / 2-\delta \leqslant\|(U+w) \Psi\| \leqslant\|U \Psi\|+\|w \Psi\| \leqslant\|U \Psi\|+w_{0}
$$

which gives the result (4.2).

Proof of theorem 1. Suppose that $H$ has an eigenvalue $E \in \Delta_{n}(B, \delta)$ with corresponding normalized eigenstate $\Psi$. From the Schwarz inequality

$$
\begin{aligned}
\|U \Psi\|^{2} & =\int_{0}^{+\infty} \mathrm{d} x \int_{-\infty}^{+\infty} \mathrm{d} y \frac{U(x)^{2}}{U^{\prime}(x)^{1 / 2}}|\Psi(\boldsymbol{r})| U^{\prime}(x)^{1 / 2}|\Psi(\boldsymbol{r})| \\
& \leqslant\left\{\int_{0}^{+\infty} \mathrm{d} x \int_{-\infty}^{+\infty} \mathrm{d} y \frac{U(x)^{4}}{U^{\prime}(x)}|\Psi(\boldsymbol{r})|^{2}\right\}^{1 / 2}\left\{\int \mathrm{d} \boldsymbol{r} U^{\prime}(x)|\Psi(\boldsymbol{r})|^{2}\right\}^{1 / 2} .
\end{aligned}
$$

We decompose the $x$ integral in the right-hand side of (5.7) into a part on $0<x<x_{0}(U)$ and $x>x_{0}(U)$. Then using

$$
\int_{0}^{x_{0}(U)} \mathrm{d} x \int_{-\infty}^{+\infty} \mathrm{d} y \frac{U(x)^{4}}{U^{\prime}(x)}|\Psi(\boldsymbol{r})|^{2} \leqslant \sup _{0 \leqslant x \leqslant x_{0}(U)}\left(\frac{U(x)^{4}}{U^{\prime}(x)}\right)
$$

and (3.11) for $x>x_{0}(U)$ we find

$$
\|U \Psi\|^{4} \leqslant A\left(E+w_{0} ; U\right)\left\langle\Psi, U^{\prime} \Psi\right\rangle
$$

where $A\left(E+w_{0} ; U\right)$ is defined in (2.1). From lemma 2 we obtain

$$
\left\langle\Psi, U^{\prime} \Psi\right\rangle \geqslant \frac{\left(B / 2-\delta-w_{0}\right)^{4}}{A\left(E+w_{0} ; U\right)} .
$$

On the other hand, from lemma $1\left\langle\Psi, U^{\prime} \Psi\right\rangle \leqslant w_{0}^{\prime}$, thus

$$
w_{0}^{\prime} \geqslant \frac{\left(B / 2-\delta-w_{0}\right)^{4}}{A\left(E+w_{0} ; U\right)} .
$$

Therefore, $H$ cannot have eigenvalues in $\Delta_{n}(B, \delta)$ as long as (2.2) holds. 


\section{Existence of spectrum}

The proof of theorem 2 is based on the auxiliary Hamiltonian (5.1). Consider a large disc of radius $R$, centred at the origin and take a smooth, radially symmetric function $g_{R}(\boldsymbol{r})$ satisfying $g_{R}(\boldsymbol{r})=1$ for $|\boldsymbol{r}| \leqslant R, g_{R}(\boldsymbol{r})=0$ for $|\boldsymbol{r}| \geqslant R,\left|\partial_{i} g_{R}(\boldsymbol{r})\right| \leqslant 1 / R, i=x, y$. Set $w_{R}=-w g_{R}$ and

$$
H_{R}=H+w_{R}=H_{0}+w\left(1-g_{R}\right) .
$$

Note that by the results of appendix $\mathrm{A} \mathrm{e}^{-t H_{R}}-\mathrm{e}^{-t H}$ is compact, hence $H_{R}$ and $H$ have the same essential spectrum ([13], chapter IV). We show that in the neighbourhood of each energy $E$ in $\Delta_{n}(B, \delta) H_{R}$, for $R$ large enough (depending on $E$ ), has a continuous spectrum. Hence because of the stability of the essential spectrum and the absence of eigenvalues, $H$ will also have a continuous spectrum around $E$.

Lemma 3. Under the assumptions of theorem 2, for any $0<\delta^{\prime}<\delta$ there exist $R_{0}\left(\delta, \delta^{\prime}\right)$ such that for all $R>R_{0}\left(\delta, \delta^{\prime}\right), H_{R}$ has no eigenvalue in $\Delta_{n}\left(B, \delta^{\prime}\right)$.

Proof. Define $w_{0}(R)=\sup _{\boldsymbol{r}}\left|w(\boldsymbol{r})\left(1-g_{R}(\boldsymbol{r})\right)\right|$ and $w_{0}^{\prime}(R)=\sup _{\boldsymbol{r}}\left|\partial_{x}\left(w(\boldsymbol{r})\left(1-g_{R}(\boldsymbol{r})\right)\right)\right|$. We have $w_{0}(R) \leqslant w_{0}$ and since $B / 2-w_{0}>\delta$,

$$
B / 2-w_{0}(R)>\delta^{\prime} .
$$

Let us now check that (2.3) is satisfied for the Hamiltonian $H_{R}$ and $\delta$ replaced by $\delta^{\prime}$. We have

$w_{0}^{\prime}(R) \leqslant \sup _{\boldsymbol{r}}\left|\partial_{x} w(\boldsymbol{r})\right|\left|1-g_{R}(\boldsymbol{r})\right|+\sup _{\boldsymbol{r}}\left|w(\boldsymbol{r}) \partial_{x}\left(1-g_{R}(\boldsymbol{r})\right)\right| \leqslant w_{0}^{\prime}+\frac{w_{0}}{R}$.

From equation (2.3)

$$
\begin{gathered}
w_{0}^{\prime}(R)<\frac{\left(B / 2-\delta-w_{0}\right)^{4}}{\sup _{0 \leqslant w_{0} \leqslant B / 2} \sup _{E \in \Delta_{n}(B, \delta)} A\left(E+w_{0} ; U\right)}+\frac{w_{0}}{R} \\
=\frac{\left(B / 2-\delta^{\prime}-w_{0}\right)^{4}}{\sup _{0 \leqslant w_{0} \leqslant B / 2} \sup _{E \in \Delta_{n}(B, \delta)} A\left(E+w_{0} ; U\right)}+\frac{w_{0}}{R} \\
+\frac{\left(B / 2-\delta-w_{0}\right)^{4}-\left(B / 2-\delta^{\prime}-w_{0}\right)^{4}}{\sup _{0 \leqslant w_{0} \leqslant B / 2} \sup _{E \in \Delta_{n}(B, \delta)} A\left(E+w_{0} ; U\right)} .
\end{gathered}
$$

We see that the third term on the right-hand side of the inequality is strictly negative for $\delta^{\prime}<\delta$ and independent of $R$. So we can find $R_{0}\left(\delta, \delta^{\prime}\right)$ large enough such that for all $R>R_{0}\left(\delta, \delta^{\prime}\right)$

$$
w_{0}^{\prime}(R)<\frac{\left(B / 2-\delta^{\prime}-w_{0}\right)^{4}}{\sup _{0 \leqslant w_{0} \leqslant B / 2} \sup _{E \in \Delta_{n}(B, \delta)} A\left(E+w_{0} ; U\right)}
$$

which implies the second inequality in (2.3) with $w_{0}$ replaced by $w_{0}(R)$ and $\delta$ replaced by $\delta^{\prime}$. As a consequence of theorem $1, H_{R}$ has no eigenvalues in $\Delta_{n}\left(B, \delta^{\prime}\right)$ for $R>R_{0}\left(\delta, \delta^{\prime}\right)$.

Lemma 4. For any $E \in \Delta_{n}(B, \delta)$ and any $\epsilon>0$ we can find $R_{1}(\epsilon, E)$ such that for $R>R_{1}(\epsilon, E)$

$$
\operatorname{dist}\left(E, \sigma\left(H_{R}\right)\right) \leqslant \epsilon .
$$

Proof. Since $\sigma\left(H_{0}\right)=\left[B / 2, \infty\left[\right.\right.$ we have $E \in \sigma\left(H_{0}\right)$. Thus there exists $\Psi_{0}$,

$$
\left\|\left(H_{0}-E\right) \Psi_{0}\right\| \leqslant \epsilon / 2 .
$$


By the triangle inequality

$\left\|\left(H_{0}+w\left(1-g_{R}\right)-E\right) \Psi_{0}\right\| \leqslant \epsilon / 2+\left\|w\left(1-g_{R}\right) \Psi_{0}\right\| \leqslant \epsilon / 2+w_{0}\left\|\left(1-g_{R}\right) \Psi_{0}\right\|$.

Since $\left\|\Psi_{0}\right\|=1$ we can find $R_{1}(\epsilon, E)$ large enough such that for $R>R_{1}(\epsilon, E)$

$$
\left\|\left(1-g_{R}\right) \Psi_{0}\right\| \leqslant \frac{\epsilon}{2 w_{0}} .
$$

From equations (5.8) and (5.9) $\left\|\left(H_{R}-E\right) \Psi_{0}\right\| \leqslant \epsilon$, and (5.6) follows from the fact that for all $\Psi,\|\Psi\|=1$, we have $\operatorname{dist}\left(E, \sigma\left(H_{R}\right)\right) \leqslant\left\|\left(H_{R}-E\right) \Psi\right\|$.

Proof of theorem 2. Take $E \in \Delta_{n}\left(B, \delta^{\prime}\right)$. We suppose that $E$ belongs to the resolvent set of $H$ and show that it leads to a contradiction. Since the resolvent is an open set we can find $\alpha>0$ such that $\operatorname{dist}(E, \sigma(H)) \geqslant \alpha$. From the fact that $H_{R}$ and $H$ have the same essential spectrum we know that $H_{R}$ could have only isolated eigenvalues in ] $E-\alpha, E+\alpha$ [, but this is impossible by lemma 3 for $R>R_{0}\left(\delta, \delta^{\prime}\right)$. Hence

$$
\operatorname{dist}\left(E, \sigma\left(H_{R}\right)\right) \geqslant \alpha \quad \text { for } \quad R>R_{0}\left(\delta, \delta^{\prime}\right) .
$$

However, if we take $\epsilon=\alpha / 2$ in lemma 4 we have

$$
\operatorname{dist}\left(E, \sigma\left(H_{R}\right)\right) \leqslant \alpha / 2 \quad \text { for } \quad R>R_{1}(\alpha / 2, E) .
$$

Therefore, we obtain a contradiction for $R>\max \left(R_{0}\left(\delta, \delta^{\prime}\right), R_{1}(\alpha / 2, E)\right)$. Thus $E \in \sigma(H)$ for all $E \in \Delta_{n}\left(B, \delta^{\prime}\right)$.

\section{Concluding remarks}

We have shown that for a large class of wall potentials (those for which the integrals (2.1) and (C.7) are finite) there are intervals of the order $B-2 w_{0}$ ( $w_{0}$ small), centred in between Landau levels, where the spectrum is continuous. This does not prevent the possibility of having a point spectrum in the vicinity of the Landau levels. In fact, it is possible to construct an attractive, impurity potential with compact support which creates a bound state with energy $B / 2-w_{0}<E<B / 2$ (recall that in this situation $B / 2$ is the infimum of the continuous spectrum of $H_{0}$ ). In the random case one expects that a dense pure point spectrum will form in the same energy interval, and that moreover for large disorder the pure point spectrum also extends above $B / 2$. In this connection see, for example, [15] for general results on band edge localization. It would be interesting to know whether this picture is valid or not near higher Landau levels.

A simpler situation is that of a homogeneous system without boundary and with crossed constant electric and magnetic fields, namely taking $U(x)=\mu x$ everywhere in space, with $\mu$ the amplitude of the electric field. Now the energy branches of $H_{0}$ are $\mathcal{E}_{n}(k)=$ $\left(n+\frac{1}{2}\right) B+(\mu / B) k-\mu^{2} / 2 B^{2}$ so that $H_{0}$ has a continuous spectrum on the whole real line. Our previous analysis shows that the spectrum of $H=H_{0}+w$ remains continuous provided that $\mu>w_{0}^{\prime}$. The absence of a point spectrum of $H$ is now obvious for $\mu>w_{0}^{\prime}$ because of (2.10). The auxiliary Hamiltonian has no eigenvalue if $\mu>w_{0}^{\prime}(R)$ (that is, in view of (5.3), for $\left.R>w_{0}\left(\mu-w_{0}^{\prime}\right)^{-1}\right)$, and has a spectrum in the neighbourhood of any energy for $R$ large enough. Then the result follows by the arguments used in theorem 2. An interesting question is the possible occurrence of a point spectrum for a weak electric field $\left(\mu<w_{0}^{\prime}\right)$. In the classical case, it is known that there exists a set of trajectories with non-zero Lebesgue measure that remain localized by the magnetic field around a hard obstacle, for non-zero but sufficiently weak electric field [16]. To our knowledge it has not been established whether this corresponds to a localized eigenstate in the quantum mechanical problem. 
Another case of a system without boundaries, but now inhomogeneous, is a given by a potential $U(x)$ that remains bounded as $x \rightarrow \infty$, for instance, the step potential

$U(x)=U_{0}\left(1-\mathrm{e}^{-\alpha x}\right) \quad x \geqslant 0 \quad \alpha>0 \quad$ and $\quad U(x)=0 \quad x \leqslant 0$.

One can check that for $U_{0}$ large enough, theorems 1 and 2 apply, for energies in between the lowest Landau levels, so that a continuous spectrum will also occur in this system. If, for example, the energy is in the interval $] B-\delta, B+\delta[$ between the first and the second levels, sufficient conditions for the occurrence of a continuous spectrum are $U_{0} \geqslant 4 B$ (so that (3.10) has a solution) and $\sqrt{U_{0}} \geqslant 2 \sqrt{2} \alpha$ (ensuring that $A(E ; U)$ in (2.1) is finite).

The notion of edge states could be made more precise by identifying them with the subspaces of continuity corresponding to the intervals $\Delta_{n}(B, \delta)$ determined in this work. This definition should be substantiated by an analysis of the spatial behaviour of the generalized eigenfunctions of $H$ in these subspaces.

\section{Appendix A. Integrable impurity potentials}

Here we show that if $w$ is an integrable impurity potential, then $\mathrm{e}^{-t H}-\mathrm{e}^{-t H_{0}}$ and $\mathrm{e}^{-t H_{R}}-\mathrm{e}^{-t H}$ are trace class.

The Feynman-Kac-Ito representation, together with the fact that $U(x)$ is positive and $w$ bounded leads to the bounds on the kernels

$$
\begin{aligned}
& \left|\left\langle\boldsymbol{r}_{1}\left|\mathrm{e}^{-s H_{0}}\right| \boldsymbol{r}_{2}\right\rangle\right| \leqslant\left\langle\boldsymbol{r}_{1}\left|\mathrm{e}^{\frac{1}{2} s \Delta}\right| \boldsymbol{r}_{2}\right\rangle \\
& \left|\left\langle\boldsymbol{r}_{1}\left|\mathrm{e}^{-s H}\right| \boldsymbol{r}_{2}\right\rangle\right| \leqslant \mathrm{e}^{s w_{0}}\left\langle\boldsymbol{r}_{1}\left|\mathrm{e}^{\frac{1}{2} s \Delta}\right| \boldsymbol{r}_{2}\right\rangle
\end{aligned}
$$

for $0 \leqslant s \leqslant t$, in terms of the free one

$$
\left\langle\boldsymbol{r}_{1}\left|\mathrm{e}^{\frac{1}{2} s \Delta}\right| \boldsymbol{r}_{2}\right\rangle=\frac{1}{2 \pi s} \mathrm{e}^{-\left|\boldsymbol{r}_{1}-\boldsymbol{r}_{2}\right|^{2} / 2 s} .
$$

If $\|\cdots\|_{H S}$ denotes the Hilbert-Schmidt norm and $f(\boldsymbol{r})$ is a square-integrable function, a direct computation using (A.1)-(A.3) gives

$$
\left\|f \mathrm{e}^{-s H_{0}}\right\|_{H S} \leqslant \frac{\|f\|_{2}}{\sqrt{4 \pi s}} \quad\left\|f \mathrm{e}^{-s H}\right\|_{H S} \leqslant \mathrm{e}^{t w_{0}} \frac{\|f\|_{2}}{\sqrt{4 \pi s}} .
$$

The trace norm of $\mathrm{e}^{-t H}-\mathrm{e}^{-t H_{0}}$ is majorized by

$$
\begin{aligned}
\left\|\mathrm{e}^{-t H}-\mathrm{e}^{-t H_{0}}\right\|_{1} & \leqslant \int_{0}^{t} \mathrm{~d} s\left\|\mathrm{e}^{-(t-s) H_{0}} w \mathrm{e}^{-s H}\right\|_{1} \\
& \leqslant \int_{0}^{t} \mathrm{~d} s\left\|\mathrm{e}^{-(t-s) H_{0}} \sqrt{|w|}\right\|_{H S}\left\|\sqrt{|w|} \mathrm{e}^{-s H}\right\|_{H S}
\end{aligned}
$$

and thus is finite by (A.4) when $w$ is integrable. The proof is the same for $\mathrm{e}^{-t H_{R}}-\mathrm{e}^{-t H}$.

\section{Appendix B. Spectrum of $H_{\omega}$}

We show that $\left[B / 2, \infty\left[\subset \sigma\left(H_{\omega}\right) \subset\left[B / 2-w_{0}, \infty[\right.\right.\right.$. The second inclusion simply follows from infspec $H_{\omega} \geqslant B / 2-w_{0}$, for all $\omega$.

For the first inclusion it is sufficient to prove that, given $E \in[B / 2, \infty[$, for any $\epsilon>0$ there exist $\Omega$ with $\operatorname{Prob}(\Omega)>0$ and $\Psi,\|\Psi\|=1$, such that $\left\|\left(H_{\omega}-E\right) \Psi\right\| \leqslant \epsilon$ for all $\omega \in \Omega$.

Since $\sigma\left(H_{0}\right)=[B / 2, \infty[$, given any $E \geqslant B / 2$ and any $\epsilon>0$, there exists a $\Psi,\|\Psi\|=1$, such that

$$
\left\|\left(H_{0}-E\right) \Psi\right\| \leqslant \epsilon / 3 .
$$


Take $\Omega=\left\{\omega|| \omega_{n m} \mid \leqslant \epsilon /\left(3 \sup _{r}|v(\boldsymbol{r})|\right)\right.$, for $\left.(n, m) \in B_{L}\right\}$, where $B_{L}$ is a square of size $2 L+1$ centred at the origin. Note that $\operatorname{Prob}(\Omega)>0$ and for all $\omega \in \Omega$

$$
\left\|V_{\omega}(\boldsymbol{r}) \Psi\right\| \leqslant \epsilon / 3+\sup _{r}|v(\boldsymbol{r})|\left\|\chi_{B_{L}^{c}} \Psi\right\|
$$

where $\chi_{B_{L}^{c}}$ is the characteristic function of the complement of $B_{L}$. Taking $L$ large enough so that the last term on the right-hand side of this inequality is less than $\epsilon / 3$ we obtain

$$
\left\|\left(H_{0}-E\right) \Psi\right\|+\left\|V_{\omega}(\boldsymbol{r}) \Psi\right\| \leqslant \epsilon .
$$

Thus by the triangle inequality $\left\|\left(H_{\omega}-E\right) \Psi\right\| \leqslant \epsilon$.

\section{Appendix C. Proof of lemma 1}

Set $D=U^{\prime}+\partial_{x} w$. The estimate (3.11) with (1.1) implies $\|D \Psi\|<\infty$. defining $R_{\lambda}=-\lambda(\mathrm{i} Y-\lambda)^{-1}, \lambda>0$, we will show below that also

$$
\left\|D R_{\lambda} \Psi\right\|<\infty
$$

and

$$
\left\|H R_{\lambda} \Psi\right\|<\infty
$$

uniformly with respect to $\lambda>0$.

Note that from (C.2), $H Y R_{\lambda} \Psi=\mathrm{i} \lambda H\left(R_{\lambda}-I\right) \Psi$ has a finite norm. Then the following identities hold:

$$
\begin{aligned}
& D \Psi=\left(R_{\lambda}-I\right) D\left(R_{\lambda}-I\right) \Psi+\left(R_{\lambda}-I\right) D \Psi+D\left(R_{\lambda}-I\right) \Psi-R_{\lambda} D R_{\lambda} \Psi \\
& R_{\lambda} D R_{\lambda} \Psi=\mathrm{i}\left(R_{\lambda} Y H R_{\lambda} \Psi-R_{\lambda} H Y R_{\lambda} \Psi\right)=-\lambda\left(H R_{\lambda} \Psi-R_{\lambda} H \Psi\right) .
\end{aligned}
$$

From (C.4) we find $\left\langle\Psi, R_{\lambda} D R_{\lambda} \Psi\right\rangle=0$ since $\Psi$ is an eigenvector of $H$. Using (C.3) we conclude that $\langle\Psi, D \Psi\rangle=0$ by letting $\lambda \rightarrow \infty$ and noting that $R_{\lambda}-I$ tends strongly to zero.

To show (C.1) and (C.2), we use the fact that $\mathrm{e}^{\mathrm{i} Y a}=\mathrm{e}^{-\mathrm{i} B y a} \mathrm{e}^{\mathrm{i} p_{x} a}$ is the operator of translations in the $x$-direction (up to a phase). This leads to the formula

$$
\left(R_{\lambda} \Psi\right)(x, y)=\lambda \int_{0}^{\infty} \mathrm{d} a \mathrm{e}^{-\lambda a} \mathrm{e}^{-\mathrm{i} B y a} \Psi(x+a, y) .
$$

Splitting $\mathrm{e}^{-\lambda a}=\mathrm{e}^{-\lambda a / 2} \mathrm{e}^{-\lambda a / 2}$ we obtain by an application of the Schwartz inequality

$$
\begin{aligned}
\int_{-\infty}^{+\infty} \mathrm{d} y\left|\left(R_{\lambda} \Psi\right)(x, y)\right|^{2} & \leqslant \lambda \int_{0}^{\infty} \mathrm{d} a \mathrm{e}^{-\lambda a} \int_{-\infty}^{+\infty} \mathrm{d} y|\Psi(x+a, y)|^{2} \\
& \leqslant C \exp \left[-\frac{x}{4 \sqrt{2}} U(x / 2)^{1 / 2}\right] .
\end{aligned}
$$

The second inequality holds for all $x \geqslant x_{0}>0$. It follows from (3.11) and the monotonicity of $U(x)$ with $C$ independent of $\lambda$. Hence, noting that $\left\|R_{\lambda}\right\|=1$, this provides the uniform upper bound with respect to $\lambda$

$$
\left\|U^{\prime} R_{\lambda} \Psi\right\|^{2} \leqslant \sup _{0 \leqslant x \leqslant x_{0}} U^{\prime}(x)+C \int_{x_{0}}^{+\infty} U^{\prime}(x)^{2} \exp \left[-\frac{x}{4 \sqrt{2}} U(x / 2)^{1 / 2}\right] .
$$

The same is also true for $\left\|D R_{\lambda} \Psi\right\|^{2}$ since $\partial_{x} w$ is bounded, so (C.1) holds. Since $Y$ commutes with $H(B)$ one has

$$
H R_{\lambda} \Psi=E R_{\lambda} \Psi+\left[U, R_{\lambda}\right] \Psi+\left[w, R_{\lambda}\right] \Psi .
$$

Using estimate (1.1) and (C.6) we find $\|U \Psi\|<\infty$ and $\left\|U R_{\lambda} \Psi\right\|<\infty$, so that all vectors in the right-hand side of (C.8) have finite norm, hence (C.2) holds. 


\section{References}

[1] Halperin B I 1982 Phys. Rev. B 252185

[2] Wen X G 1991 Phys. Rev. B 4311025

[3] Froehlich J and Kerler T 1991 Nucl. Phys. B 354369

[4] For a review with a complete list of references see Froehlich J and Studer U M 1993 Rev. Mod. Phys. 65733

[5] Chang A M, Pfeiffer L N and West K W 1996 Phys. Rev. Lett. 772538 See also 1996 Phys. Today 4919

[6] Dorlas T C, Macris N and Pulé J V 1997 J. Stat. Phys. 87847

Dorlas T C, Macris N and Pulé J V 1998 Characterization of the spectrum of the Landau Hamiltonian with delta impurities Commun. Math. Phys. to appear

Dorlas T C, Macris N and Pulé J V 1998 Quantum Hall effect without divergence of the localization length to appear

[7] Dorlas T C, Macris N and Pulé J V 1995 Helv. Phys. Acta 68330

Dorlas T C, Macris N and Pulé J V 1996 J. Math. Phys. 371574

[8] Combes J M and Hislop P D 1996 Commun. Math. Phys. 177603

[9] Wang W M 1997 J. Funct. Anal. 1461

[10] Froehlich J and Graf Private communication

Walcher J 1998 Anwendung positiver kommutatoren auf den quanten Hall effect Diploma Work ETHZ

[11] Reed M and Simon B 1978 Methods of Modern Mathematical Physics vol 2 (New York: Academic)

[12] Reed M and Simon B 1978 Methods of Modern Mathematical Physics vol 4 (New York: Academic)

[13] Kato T 1980 Perturbation Theory for Linear Operators 2nd edn (Berlin: Springer)

[14] Simon B 1979 Functional Integration and Quantum Physics (New York: Academic)

[15] Barbaroux J M, Combes J M and Hislop P D 1997 Helv. Phys. Acta 7016

[16] Berglund N, Hansen A, Hauge E H and Piasecki J 1996 Phys. Rev. Lett. 772149 